\title{
Pain description and presentation in children admitted to a teaching hospital in Ghana
}

\begin{abstract}
Background/Aim: Pain is a cardinal symptom of many disease states and a common reason for hospital attendance. In children, however, it is often undertreated, partly due to the difficulty in its assessment. This study outlines the causes, anatomic locations and verbal descriptions of pain in children admitted to the Pediatric Emergency Unit of the Komfo Anokye Teaching Hospital in Ashanti, Ghana.
\end{abstract}

Methods: This is a cross-sectional study of 273 children aged 5-12 years. Using a structured questionnaire, we obtained information through patient interview and folder review on the causes, locations and verbal descriptions of pain in children hospitalized for non-traumatic medical conditions including cancers.

Findings: Sickle cell disease, infections and cancers were the main causes of pain in children admitted to the unit. Abdominal pain was the most frequently reported. Pain description in either English or the local language, Akan was difficult for most children.

Conclusion/Recommendations: Pain description is difficult for most Ghanaian children as it requires the use of complex adult language. Therefore, in the management of pain in this population, it is recommended that the clinician places less emphasis on the exact description of pain and rather focus on effective pain relief.

Keywords: pain, descriptions, presentation, children, Akan, Ghanaian
Volume 2 Issue 4 - 2015

\author{
Hammond CK,' Dogbe J, ',2 Paintsil V,' Osei- \\ Tutu L,' Owusu M³ \\ 'Department of Child Health, Komfo Anokye Teaching Hospital, \\ Ghana \\ ${ }^{2}$ Kwame Nkrumah University of Science and Technology, Ghana \\ ${ }^{3}$ Kumasi Centre for Collaborative Research, Ghana
}

\begin{abstract}
Correspondence: Joslin Dogbe, Department of Child Health, Kwame Nkrumah University of Science and Technology and the Komfo Anokye Teaching Hospital, P.O. Box 1934, Kumasi, Ghana,
\end{abstract} Tel 233-24427I70I, Email slimdogbe@gmail.com

Received: March 13,2015 | Published: May 19, 2015
Abbreviations: ARI, acute respiratory infection; CHRPE, committee on human research and publication ethics; CI, confidence interval; JHS, junior high school; KATH, Komfo Anokye teaching hospital; KNUST, Kwame Nkrumah university of science and technology; MSLC, middle school leaving certificate; PEU, pediatric emergency unit; PI, principal investigator; SCD, sickle cell disease; SD, standard deviation; SHS, senior high school; VOC, vasoocclusive crisis; WHO, world health organization; Yr, year(s)

\section{Introduction}

Pain is a cardinal symptom of many disease states and one of the common reasons people seek medical attention. Many people know what pain can feel like, but it is still difficult to define. It is a complex perception that differs enormously among individual patients, even those who appear to have identical injuries or illnesses.

Advances in pain research have resulted in a remarkable ability to understand this complex perception and to keep it in check. Today, the relief of pain is considered a human right and there is a heightened awareness of pain as the "fifth vital sign" that should be monitored with the same vigilance as blood pressure, pulse, temperature and respiratory rate. ${ }^{1}$ It is now general knowledge that proper assessment of pain in terms of its causes, description and severity are very important for appropriate and adequate management leading to quicker clinical recovery, shorter hospital stay, fewer re-admissions and improved quality of life.

Children suffer pain from medical illnesses, trauma, hospital procedures, cancers and non-malignant chronic conditions. ${ }^{2-4}$ Among the medical conditions, infections and inflammations are important sources of pain. The sick Ghanaian child may experience headache from malaria or meningitis, neck pain from meningitis, chest pain from pneumonia and abdominal pain from gastroenteritis, urinary tract infections, enteric fever or acute hepatitis. He or she may also experience pain in the extremities from osteomyelitis or septic arthritis.

Besides infections, the West African child may also suffer pain from sickle cell disease (SCD). In Ghana, SCD is a significant public health burden, affecting $2 \%$ of newborns and presenting a unique pain management challenge in paediatric practice. ${ }^{5,6} \mathrm{In} \mathrm{SCD}$, episodic micro vascular occlusions occurring at one or many sites often lead to pain, local inflammation and disability. This is referred to as the vasoocclusive crisis (VOC). Sickle cell VOC can occur in any part of the body but is commonly seen within the long bones of the extremities, the ribs, sternum, spine and pelvic bones. ${ }^{7,8} \mathrm{VOC}$ has been reported as the single most important reason for frequent emergency department visits in SCD, accounting for about $79-91 \%$ of emergency department visits and about $59-68 \%$ of all admissions. Pain intensity ratings in hospitalized children with sickle cell disease are persistently reported at moderate or severe levels. ${ }^{9}$

Cancers also constitute other important sources of pain in sick children. Among the many childhood cancers seen at the Komfo Anokye teaching Hospital (KATH) are lymphomas, acute leukaemias, nephroblastomas, neuroblastomas, rhabdomyosarcomas and retinoblastomas..$^{10}$ In cancers, there are three main sources of pain: pain from tumour invasion, pain from therapy and psychological pain. ${ }^{11-13}$ Miser et al. ${ }^{12}$ reported that among 139 children hospitalized for cancers and evaluated over a six-month period, a third of the patients had pain as a result of tumour invasion, while over $40 \%$ had pain resulting from therapy and procedures. ${ }^{12}$

In a survey of the fellows of the West African College of Surgeons attending the Annual Scientific Conference in Accra, Ghana in 1996 , cancer pain was thought by $52 \%$ of the fellows to be due to the cancer and its treatment while $47 \%$ thought it was due to the 
cancer and the fear of dying. The respondents estimated that $70-90 \%$ of their patients who included both children and adults had severe pain at presentation. ${ }^{13}$ Trauma is another source of pain in children. This includes accidental injuries such as heat burns, chemical burns, soft tissue injuries and fractures. Children are also victims of nonaccidental injuries sustained from abuses. ${ }^{2,4}$ Hospitalized children also suffer acute pain from both diagnostic and therapeutic procedures on a daily basis. Common among hospital procedures that cause pain in children are blood-letting, intravenous cannulations, intramuscular injections, lumber punctures, wound care and biopsies. Surgery, both major and minor, are important sources of pain in all patients. ${ }^{4,14}$

In adults, several verbal descriptors have often been used to assess the quality of pain. In a descriptive study to identify words that American children use to describe their pain, Wilkie et al. ${ }^{15}$ identified a list of 56 descriptors used by school-age and adolescent Americans. ${ }^{15}$ This list of descriptors was made up of 37 sensory words, 11 affective words and 8 evaluative words describing the qualities of their pain experience. Similar studies to look at the words used by Dutch, ${ }^{16}$ Canadian, ${ }^{17}$ Arab, ${ }^{18}$ Asian-American and Latin-American ${ }^{19}$ children to describe their pain have been published. In 1989, Verkes et al., ${ }^{16}$ examined the semantic structure and the underlying dimensions of 176 common Dutch words used to describe various kinds of pain. ${ }^{16}$ Results from this study strongly indicate a cross-cultural generality in the structure of pain descriptions. A report by Finley et al., ${ }^{17}$ also shows little evidence that pain perception is modified by cultural or ethnic factors. ${ }^{17}$ The researchers however point out that pain expression by children and interpretation by adult caregivers may be affected by the culture of the patient or the caregiver. Also, spirituality and religion are known to have a substantial impact on patients' understanding of pain and decisions about pain management, as reported by Unruh AM. $^{20}$

Gender role differentiation in the perception of pain has been reported in most cultures. Generally, girls are considered docile, dependent and emotional, whereas boys are considered assertive, brave and strong. Abu-Saad ${ }^{18}$ reported that Asian and Arab girls more frequently identified psychological causes and used more affective and evaluative words for pain than boys. ${ }^{18}$ Arab girls were found to use words like "sad", "embarrassed" and "feels like running away" while boys used words like "brave", "angry" and "don't cry". Differences among boys and girls in the frequency of pain reporting have been studied with conflicting reports. While some studies report no sex differences in children's pain reporting and pain severity, others have demonstrated a higher frequency of reporting among girls than boys. In a cross-sectional survey of all surgical and medical inpatients in a Canadian tertiary paediatric hospital, Taylor et al., ${ }^{14}$ reported no sex difference in the frequency of pain reporting or the intensity of pain reported. ${ }^{14}$ This was in keeping with previous Canadian publications by Cummings et al., ${ }^{2}$ and Johnston et al. ${ }^{21}$

However, studies among Dutch children and adolescents have provided contrary reports. In a cross-sectional population survey of Dutch children aged up to 18 years, Perquin et al., ${ }^{22}$ reported that except for younger children aged less than 3 years, girls reported pain more frequently than boys. Gender differences were significantly particular in adolescents. ${ }^{22}$ Another Dutch study by Merlijn et al., ${ }^{23}$ showed similar findings. Some children may not report pain even when present. Thus pain prevalence is not necessarily equal to pain reporting. A critical review of the above publications however shows that a clear distinction is not drawn between the frequency of pain reporting and pain prevalence among the sexes.
Gender-specific differences have also been reported in the perceived triggers of pain in children. Among the 749 children and adolescents studied by Roth-Isigkeit et al., ${ }^{24}$ the self-reported triggers for pain varied between girls and boys. Girls stated more often than boys that their pain was triggered by weather conditions, illness, anger, disputes, family conditions and sadness. ${ }^{24}$ In contrast, boys stated more often than girls that their pain was triggered by physical exertion. To better understand what the Ghanaian child refers to as pain, we undertook a cross-sectional study to determine the causes of pain in children, the anatomic locations of pain and the various factors that influence children's ability to describe pain.

\section{Materials and methods}

This is a cross-sectional study involving 273 children aged 5-12 years who were admitted to the Pediatric Emergency Unit (PEU) of KATH from March 2012 to July 2012. The PEU, with a bed capacity of twenty, runs a 24 hour emergency service and admits patients aged 3 months to 12 years who are presenting with pediatric medical emergencies. On the average, patients stay at the unit for 24 hours after which they are either transferred to the main pediatric wards or discharged home.

Ethical approval for the study protocol was obtained from the Committee on Human Research and Publications Ethics (CHPRE) of the Kwame Nkrumah University of Science and Technology.

\section{Selection of subjects}

Folders of all patients admitted to the PEU during the study period were examined by the investigators for complaints of pain. Patients aged 5-12 years who reported pain as a presenting complaint or admitted to pain on direct questioning were included in the study. Patients were excluded if their pain was thought to be due to trauma, post-operative or hospital procedure. Also, patients with impaired consciousness or cognitive impairment were excluded. Patients were also excluded if the parents refused consent to join the study.

\section{Tools and methods of data collection}

A structured and verbally administered questionnaire with pre-coded responses was used to obtain information on the child's demographic details, the anatomic location of the child's pain and the child's description of his/her pain in English or the Ghanaian local language (Akan).

On the child's demographic characteristics, the information obtained were the child's age in years, gender, his or her primary language (i.e. the language that the speaks and understands at home) and his or her school grade classified as preschool, lower primary (first to third grade), upper primary (fourth to sixth grade) or junior high (seventh to ninth grade).

Next, the child was asked the exact location(s) of his or her pain. They were then asked to describe their pain in their primary language. For children with multiple pain locations, they were asked to describe the worst pain. Finally, the patient's admission notes were reviewed and the admission diagnosis was recorded as the cause of pain. Data was collected during the day from $8 \mathrm{am}$ to $8 \mathrm{pm}$ and on all days during the four mouth study period.

\section{Data analysis}

Using Epi Info 3.5.1, the questionnaire was transformed into an electronic case record form. The data was entered and stored as a Microsoft Access document. It was then analyzed using Stata 8.0. 
In the analysis, the ages were grouped as 5-7 years, 8-10 years and 11-12 years. The school grades were also grouped as not in school, lower grades (preschool and lower primary) and upper grades (upper primary and junior high).

\section{Results and discussion}

\section{Results}

Demographic characteristics of patients: The demographic characteristics of the respondents are as shown in table 1. In summary, participants were aged 5-12 years with a mean age of 8.51 years $(\mathrm{SD}=2.28,95 \% \mathrm{CI}=8.24-8.78)$. There were $143(52.4 \%)$ males and $130(47.6 \%)$ females. All but $5(1.8 \%)$ of the children were in school (Table 1).

Table I Participant demographic characteristics

\begin{tabular}{ll}
\hline Variable & Distributions (n=273) \\
\hline Age & \\
Mean (SD) & $8.5 \mathrm{I}(2.28)$ \\
Gender & \\
Male & $\mid 43(52.4 \%)$ \\
Female & $130(47.6 \%)$ \\
School Grade & \\
Not In School & $5(1.8 \%)$ \\
Preschool & $5 \mathrm{I}(18.7 \%)$ \\
Lower Primary & $124(45.4 \%)$ \\
Upper Primary & $86(31.5 \%)$ \\
Junior High & $7(2.6 \%)$ \\
Primary Language & \\
Akan & $199(72.9 \%)$ \\
English & II (4.0\%) \\
Other & $63(23.1 \%)$ \\
\hline
\end{tabular}

Description of pain in the local ghanaian language: In order to understand the Ghanaian context of pain description, we first aggregated and translated corresponding Ghanaian language (Akan) interpretation of pain in English It is worth noting that several English pain descriptors could be translated into a single Akan word or phrase. Also, these Akan descriptors could have multiple meaning in English depending on the context in which they are used and also on the location of the pain. Table 2 shows some English pain descriptors and their closest Akan translations.

Causes of pain: The main causes of pain were SCD, cancers and infections. Ninety-seven (35.5\%) patients had SCD-related pain, while $47(17.2 \%)$ and 94 (34.5\%) had cancers and infection-related pain respectively. Other causes of pain were reported in $35(12.8 \%)$ patients (Figure 1).

The specific causes of pain in SCD, cancers and infections are outlined in Table 3. Vaso-occlusive crisis (VOC) was the commonest cause of pain in SCD patients accounting for $61.9 \%$ of all SCDrelated pain. Among patients with suspected cancers, lymphoma was the most common diagnosis accounting for $57.4 \%$. Key among the infectious causes of pain was acute respiratory infections, meningitis, enteric fever and malaria accounting for $19.2 \%, 13.8 \%, 13.8 \%$ and $10.6 \%$ patients respectively (Table 3 ).

Anatomic locations of pain: Abdominal pain was the most frequently reported accounting for $26.7 \%$ of patients. This was followed by head and neck pain, lower extremity, chest, upper extremity, back and pelvic pain. Forty-one (15\%) patients reported pain in multiple locations. This is illustrated in Figure 2.

Table 2 Akan pain words and equivalent English words

\begin{tabular}{ll}
\hline $\begin{array}{l}\text { Twi Pain } \\
\text { Words }\end{array}$ & Equivalent English Pain Descriptors \\
\hline Etutu & Beating, Hitting, Pounding, Punching, Throbbing, Shooting \\
Ehyehye & Burning, Sore-Like, Hot, Blistering, Sting-Like \\
Ewっ & Stabbing, Sharp, Cutting, Biting, Splitting, Pin-Like \\
Ewuwə & Pin-Like, Colicky, Pinching, Sharp \\
Edwa & Aching, Cramping, Hurting, Crushing, Shooting \\
Ekeka & Itching, Scratching, Biting \\
Titiritii & Numbness, Stiff \\
Efemfem & Tingling, Pinching \\
Bawee & Stiff,Tight, Pressure, Numb, Cramping \\
Dudruduruu & Numb, Stiff, Tight, Pressure, Swollen \\
\hline
\end{tabular}

Table 3 Specific causes of pain

\begin{tabular}{|c|c|c|}
\hline Disease/Condition & Frequency & Percentage \\
\hline \multicolumn{3}{|l|}{ Scd-Related Pain } \\
\hline Vaso-Occlusive Crisis & 60 & 61.9 \\
\hline Acute Chest Syndrome & 7 & 7.2 \\
\hline Mesenteric Pain & 12 & 12.3 \\
\hline Osteomyelitis & 8 & 8.2 \\
\hline Septic Arthritis & 5 & 5.2 \\
\hline Splenic Sequestration & 3 & 3.1 \\
\hline Priapism & 2 & 2.1 \\
\hline Total & 97 & 100 \\
\hline \multicolumn{3}{|l|}{ Cancer-Related Pain } \\
\hline Lymphoma & 27 & 57.4 \\
\hline Leukaemia & 9 & 19.1 \\
\hline Abdominal Malignancy (Non-Specific) & 3 & 6.4 \\
\hline Rhabdomyosarcoma & 2 & 4.3 \\
\hline Hepatoma & 2 & 4.3 \\
\hline Other Tumours & 4 & 8.5 \\
\hline Total & 47 & 100 \\
\hline \multicolumn{3}{|l|}{ Infection-Related Pain } \\
\hline Malaria & 10 & 10.6 \\
\hline Meningitis & 13 & 13.8 \\
\hline Acute Respiratory Infections & 18 & 19.2 \\
\hline Enteric Fever & 13 & 13.8 \\
\hline Urinary Tract Infections & 7 & 7.4 \\
\hline Hepatitis & 5 & 5.3 \\
\hline Cellulitis (Extremities) & 6 & 6.4 \\
\hline Cellulitis (Facial) & 4 & 4.3 \\
\hline $\begin{array}{l}\text { Osteomyelitis/Septic Arthritis (Non- } \\
\text { SCD) }\end{array}$ & 4 & 4.3 \\
\hline Other Infections & 14 & 14.9 \\
\hline Total & 94 & 100 \\
\hline
\end{tabular}


Table 4 Patients demographic characteristics vs ability to describe pain

\begin{tabular}{|c|c|c|c|}
\hline \multirow{2}{*}{ Variables } & \multicolumn{2}{|c|}{ Ability to describe pain } & \multirow{2}{*}{ P-Value } \\
\hline & Able to describe (\%) & Unable to describe (\%) & \\
\hline \multicolumn{4}{|l|}{ Age (yrs) } \\
\hline $5-7$ & $6(5.8)$ & $98(94.2)$ & \multirow{4}{*}{0} \\
\hline $8-10$ & $42(41.6)$ & $59(58.4)$ & \\
\hline $11-12$ & $50(73.5)$ & $18(26.5)$ & \\
\hline Total & $98(35.9)$ & $175(64.1)$ & \\
\hline \multicolumn{4}{|l|}{ Sex } \\
\hline Male & $49(34.3)$ & $94(65.7)$ & \multirow{3}{*}{0.134} \\
\hline Female & 49 (37.7) & $81(62.3)$ & \\
\hline Total & 98 (35.9) & $175(64.1)$ & \\
\hline \multicolumn{4}{|l|}{ School grade } \\
\hline Not in school & I (20.0) & $4(80.0)$ & \multirow{4}{*}{0} \\
\hline Lower grades & $34(19.4)$ & $|4|(80.6)$ & \\
\hline Upper grades & $63(67.7)$ & $30(32.3)$ & \\
\hline Total & 98 (35.9) & $175(64.1)$ & \\
\hline \multicolumn{4}{|c|}{ Primary language } \\
\hline Akan & $80(40.2)$ & $119(59.8)$ & \multirow{4}{*}{0.768} \\
\hline English & $4(36.4)$ & $7(63.6)$ & \\
\hline Others & $14(22.2)$ & $49(77.8)$ & \\
\hline Total & 98 (35.9) & $175(64.1)$ & \\
\hline
\end{tabular}



Figure I General causes of pain.

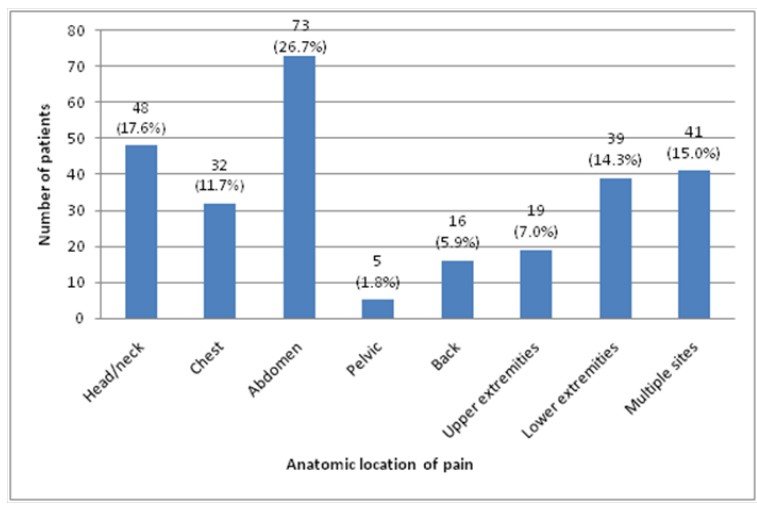

Figure 2 Anatomic locations of pain.

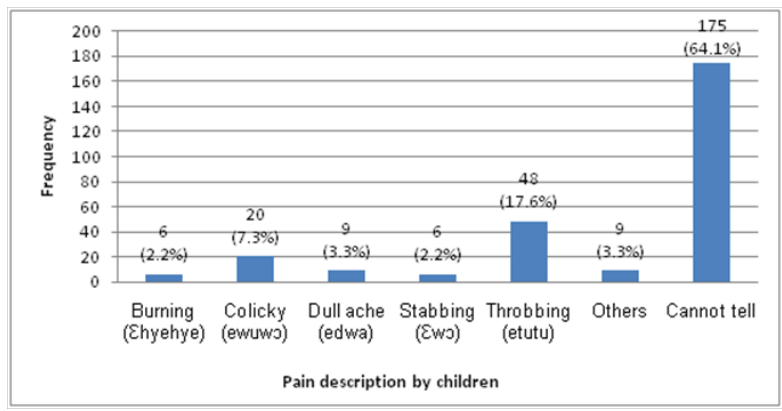

Figure 3 Pain descriptions by children.

Pain descriptions by children: Figure 3 outlines the pain descriptions used by the children and the frequency of usage. One hundred and seventy-five children $(64.1 \%)$ could not describe their pain using English or Akan pain descriptors. Only 98 (35.9\%) children could describe their pain. Common descriptions were throbbing (etutu), colicky (ewuwつ), dull ache (edwa), burning (chyehye) and stabbing pain (Ewכ). Nine (3.3\%) patients used other descriptors such as numbness (titiritii), stiff (bawee), itching (Ekeka) or tight (dudruduruu), or long sentences rather than descriptive words or phrases. In children who reported multiple pain locations, the site with the worst pain was described.

Children's demographic characteristics Versus Ability to describe pain: Children's ages, sex, school grades and primary languages were cross-tabulated with their ability to describe pain and the results are as shown in Table 4. 
The ages were grouped as 5-7, 8-10 and 11-12 years. The percentage of children able to describe their pain increased progressively with age with a corresponding decrease in the percentage unable to describe their pain ( $\mathrm{p}$-value $=0.000)$.

The results also show that more females $(37.7 \%)$ were able to describe their pain as compared to males $(34.3 \%)$. The difference is however not statistically significant $(\mathrm{p}$-value $=0.134)$.

In relation to school grades, only $19.4 \%$ of children in lower grades were able to describe their pain compared to $66.7 \%$ in upper grades $(\mathrm{p}$-value $=0.000)$.

The patients' ability to describe pain was also assessed in relation to their primary language. Among the Akan-speaking children, $40.2 \%$ were able to describe their pain as compared to $36.4 \%$ Englishspeaking children and $22.2 \%$ non-Akan non-English speaking children. This was however not statistically significant $(\mathrm{p}$-value $=$ $0.768)$.

\section{Discussion}

Demographic characteristics: Children were aged $5-12$ years with a median age of 8 years and a male to female ratio of 1.1:1. This suggest a fairly equal gender distribution in support of the assertions by Taylor et al., ${ }^{14}$ who reported that there are no significant sex differences in the prevalence of pain in paediatric in-patients. Cummings et al., ${ }^{2}$ and Johnston et al., ${ }^{21}$ have also reported that there are no significant sex differences in the prevalence of pain and in the frequency of pain reporting in hospitalized children. It is interesting to note that all three studies cited above are from Canadian teaching hospitals. ${ }^{2,14,21}$ In contradiction, two Dutch studies by Perquin et al., ${ }^{22}$ and Merlijn et al., ${ }^{23}$ reported a higher frequency in pain reporting in girls.

In $72.9 \%$ of the respondents, Twi was the primary language used at home. This reflects the dominance of Akan-speaking tribes in Ashanti and the adjoining regions. However, there are still families within the catchment areas of KATH who are non-Akan speaking. With these families, interviews during hospital visits at KATH are difficult. In some instances, they may speak and understand English or some form of Akan language. Otherwise, an interpreter was needed to conduct the interview. In the description of pain, this presented a challenge to both the interviewer and the respondent.

Almost all children recruited were in school at different grades from preschool to junior high school. Among the many factors that have increased school enrollment in Ghana in recent times are the capitation grants, improved accessibility, the school feeding programme, and the provision of free school uniforms and textbooks.

Causes of pain: Pain in sickle cell disease was the most frequently reported. VOC was the commonest cause of SCD-related pain accounting for $61.9 \%$ of SCD respondents. This compares well with findings by Jacob \& Mueller ${ }^{9}$ who reported that VOC is the single most important reason for emergency department visits in SCD, accounting for about 59-68\% of all SCD admissions. Other important causes of pain in SCD that were seen at the PEU were acute chest syndrome, mesenteric pain, bone and joint infections, splenic sequestration and priapism. ${ }^{9}$

Next to SCD as the commonest cause of pain were infections. Over a third of the study subjects suffered pain from various infections, notably acute respiratory infections (ARIs), meningitis, enteric fever and malaria; altogether accounting for about $60 \%$ of the infectionrelated pain. This reflects the trend of infections commonly seen at the PEU during the period March to July when the dry harmattan season gives way to the rainy season. In the dry season before the onset of the rains, ARIs and meningitis are commonly seen. In the months of June and July when the rains set in, malaria becomes the most common infection diagnosed at the PEU. ${ }^{10}$

Cancers were reported as the third common causes of pain in children admitted to the emergency unit. Even though these were provisional diagnosis, they accounted for $17.2 \%$ of pain admissions. Over $57 \%$ of these suspected cancers were thought to be lymphomas. Next to lymphomas were leukaemias, accounting for a little less than one-fifth of cancer pain. Provisional diagnoses were made for several other tumours but their total contribution to cancer pain was less than $25 \%$. This suggests that lymphomas in children continue to be an important malignancy seen at KATH. In a region where malaria remains endemic, it is very likely that a large majority of these tumors were Burkitt lymphoma, an assertion supported by existing data on childhood cancers at KATH. ${ }^{10}$

Locations and descriptions of pain: The abdomen was the commonest anatomic location for pain in over one-quarter of respondents. Head and neck, lower extremities, chest, upper extremities, back and pelvic pains followed in decreasing frequency. Pain locations may vary from one study to the other depending on the subjects and the disease entity being studied. In children and adolescents, head/neck and abdominal pain have been reported with the highest frequency., ${ }^{42}$ However, in a cohort of 79 adult patients living with HIV/AIDS seen at the antiretroviral clinic in a Nigerian tertiary hospital, Wahab KW \& Salami $\mathrm{AK}^{25}$ reported a higher frequency of lower limb pain followed by head and neck and abdominal pain. ${ }^{25}$

Although many children in this study located their pain to a specific anatomic region, pain in multiple locations were reported in $15 \%$ of patients. In these children, although the pain descriptions were obtained from the site worst affected, pain from other locations could influence their response.

Over $64 \%$ of children could not describe their pain using Akan or English pain descriptors. This reaffirms the earlier assertion that pain descriptive words are mostly adult language and not easy to use by children. Of those who could describe their pain, descriptions were mainly throbbing (etutu), colicky (ewuwə), dull ache (edwa), burning (chyehye) and stabbing ( $(\mathrm{w})$ ) in decreasing frequency. A few other patients used numbness (titiritii), stiff (bawee), itching (ckeka), tight (dudruduruu), or long sentences to describe their pain. As reported in earlier studies by Beyer and Wells ${ }^{19}$ Verkes et al., ${ }^{16}$ Finley et al., ${ }^{17}$ and Abu-Saad, ${ }^{18}$ accurate pain description is always a challenge for the young child and even for some adolescents and adults. As such, it is not surprising that nearly two-thirds of patients in this study could not use any of the pain descriptive words.

Patients' demographic characteristics Versus Ability to describe pain: The inability of children in this study to use pain language was worse at younger ages. In children 5-7 years old, $94.2 \%$ could not describe their pain while at $11-12$ years; only $26.5 \%$ could not describe their pain. Statistical analysis of this result suggest that age is significantly associated with a child's ability to describe the nature of pain ( $p$-value $=0.000$ ). As children age beyond 8 years, they begin to acquire adequate vocabulary to describe a phenomenon as complex as pain. This supports the findings by Wilkie et al., ${ }^{15}$ in their study to determine the validity and reliability of children's and adolescents' pain language. ${ }^{15}$

In this study, the percentages of male and female children unable to describe the nature of their pain were almost equal (65.7\% vs.62.3\%) with no significant difference $(\mathrm{p}=0.134)$. This is in keeping with 
the findings from a Dutch study by Verkes et al., ${ }^{16}$ in which no sex differences were seen in the use of pain descriptive words. In this Dutch study, however, the respondents were adults and the focus was to rate the intensity of pain using descriptive words rather than a scale. ${ }^{16}$

In terms of school grades, only $19.4 \%$ of children in preschool and lower primary were unable to use the pain descriptive words, in contrast to $67.7 \%$ of children in upper primary and junior high schools. This was also statistically significant $(\mathrm{p}$-value $=0.000)$ and can be explained in terms of the child's age since children generally move up in school grades as they age.

Children's ability to describe their pain was not significantly related to their primary language. Across all language groups (Akan, English and others); the majority $(59.8 \%, 63.6 \%$ and $77.8 \%$ respectively) of children were unable to describe the nature of their pain ( $p$-value $=0.768$ ). Even though not statistically significant, this percentage was higher for the non-Akan non-English speaking children and this could be attributed to the use of interpreters during the interviews. The cross-cultural generality in the structure of pain description by children has already been reported by Finley et al., ${ }^{17}$ in a review article. However, it is worth noting that the writers in this review article examined various aspects of culture including race, ethnicity, religion, customs and beliefs, and not just the language. Though pain description by children was not so different in the various languages, the individual beliefs and attitudes of caregivers affected how the pain is reported, as noted by Finley et al. ${ }^{17}$ It is therefore important for clinicians to be sensitive to the caregiver's beliefs and attitudes in assessing the nature of pain in any sick child. ${ }^{17}$

\section{Conclusion and recommendations}

This study has shown that among children admitted to the Pediatric Emergency Unit (PEU) of the Komfo Anokye Teaching Hospital, there are no significant gender differences in the frequency of pain reporting. Sickle cell disease, infections and cancers are the leading causes of pain in children admitted to the emergency unit. About two-thirds of children $(64.1 \%)$ were unable to describe their pain using Akan or English pain descriptors. Among the few who could describe their pain, the common descriptions used were throbbing (etutu), colicky (ewuwə), dull ache (edwa), burning (chyehye) and stabbing ( $\varepsilon w \supset$ ). This study has also shown that the gender and the primary language of Ghanaian children do not influence their ability or inability to describe pain. However, this ability is influenced by their ages and school grades. For children in pain, we recommend that clinicians and therapists should place less emphasis on the exact description and rather focus on effective relief of their pain.

\section{Acknowledgments}

None.

\section{Conflicts of interest}

Authors declare that there is no conflict of interest.

\section{References}

1. Phillips DM. JCAHO pain management standards are unveiled. Joint Commission on Accreditation of Healthcare Organizations. JAMA. 2000;284(4):428-429.

2. Cummings EA, Reid GJ, Finley GA, et al. Prevalence and source of pain in pediatric inpatients. Pain. 1996;68(1):25-31.
3. Kortesluoma RL, Nikkonen M, Serlo W. "You just have to make the pain go away" -children's experiences of pain management. Pain Manag Nurs. 2008;9(4):143-149.

4. Loizzo A, Loizzo S, Capasso A. Neurobiology of pain in children: an overview. Open Biochem J. 2009;3:18-25.

5. Ohene-Frempong K, Joseph O, Hannah T, et al. Screening newborns for sickle cell disease in Ghana. Pediatrics. 2008;121(Suppl 2):S120-S121.

6. Kyerewaa Edwin A, Edwin F, Etwire V. Controlling Sickle Cell Disease in Ghana-ethics and options. Pan Afr Med J. 2011;10:14.

7. Adekile AD, Adeodu OO. Haemoglobinopathies. Paediatrics and Child Health in a tropical region. African Educational Services: Owerri, Nigeria; 2007. 373-390 p.

8. DeBaun MR, Vichinsky E. Hemoglobinopathies: Nelson Textbook of Pediatrics. 18th ed. Saunders: Philadelphia, London; 2007.

9. Jacob E, Mueller BU. Pain experience of children with sickle cell disease who had prolonged hospitalizations for acute painful episodes. Pain Med. 2008;9(1):13-21.

10. KATH. Annual Report 2009.

11. Amery J. Children's palliative care in Africa. Oxford University Press: London; 2009. 2-444 p

12. Miser AW, Dothage JA, Wesley RA, et al. The prevalence of pain in a pediatric and young adult cancer population. Pain. 1987;29(1):73-83.

13. Soyannwo A, Amanor-Boadu SD. Management of cancer pain--a survey of current practice in West Africa. Niger Postgrad Med J. 2001;8(4):175-178

14. Taylor EM, Boyer K, Campbell FA. Pain in hospitalized children: a prospective cross-sectional survey of pain prevalence, intensity, assessment and management in a Canadian pediatric teaching hospital. Pain Res Manag. 2008;13(1):25-32.

15. Wilkie DJ, Holzemer WL, Tesler MD, et al. Measuring pain quality: validity and reliability of children's and adolescents' pain language. Pain. 1990;41(2):151-159.

16. Verkes RJ, Van der Kloot WA, Van der Meij J. The perceived structure of 176 pain descriptive words. Pain. 1989;38(2):219-229.

17. Finley, G Allen, Kristjánsdóttir $\mathrm{O}$, et al. Cultural influences on the assessment of children's pain. Pain Res Manag. 2009;14(1):33-37.

18. Abu-Saad H. Cultural group indicators of pain in children. Matern Child Nurs J. 1984;13(3):187-196.

19. Beyer J, Wells N. Assessment of cancer pain in children. Cancer Pain, Lippincott-Raven: Philadelphia, USA; 1993. 57-84 p.

20. UnruhAM. Spirituality, religion, and pain. Can JNurs. 2007;39(2):66-86.

21. Johnston CC, Abbott FV, Gray-Donald K, et al. A survey of pain in hospitalized patients aged 4-14 years. Clin J Pain. 1992;8(2):154-163.

22. Perquin CW, Hazebroek-Kampschreur AA, Hunfeld JA, et al. Pain in children and adolescents: a common experience. Pain. 2000;87(1):51-58.

23. Merlijn VP, Hunfeld JA, van der Wouden JC, et al. Psychosocial factors associated with chronic pain in adolescents. Pain. 2003;101(1-2):33-43.

24. Roth-Isigkeit A, Thyen U, Stoven H, et al. Pain among children and adolescents: restrictions in daily living and triggering factors. Pediatrics. 2005;115(2):e152-e162.

25. Wahab KW, Salami AK. Pain as a Symptom in Patients Living With HIV/AIDS Seen at the Outpatient Clinic of a Nigerian Tertiary Hospital. J Int Assoc Physicians AIDS Care (Chic). 2011;10(1):35-39. 